\title{
A INFLUÊNCIA DA CINESIOTERAPIALABORAL NA QUALIDADE DE VIDA DOS PROFESSORES E NO PROCESSO DE ENSINO APRENDIZAGEM
}

Elgison da Luz dos SANTOS ${ }^{1}$

Carla Daniela CÂMARA²

\begin{abstract}
${ }^{1}$ Fisioterapeuta com especialização em Ensino de Ciências pela Universidade Tecnológica Federal do Paraná - (Campus Medianeira), elgisantos20@gmail.com.

${ }^{2}$ Doutora em Engenharia Hidráulica e Saneamento pela Universidade de São Paulo. Professora da Universidade Tecnológica Federal do Paraná (Campus Medianeira), camara@utfpr.edu.br.
\end{abstract}

Recebido em: 05/05/2016 - Aprovado em: 22/11/2017 - Disponibilizado em: 30/12/2017

\begin{abstract}
RESUMO:
A cinesioterapia Laboral (CL) pode ser entendida como uma série de exercícios físicos elaborados no próprio ambiente de trabalho, visando à qualidade de vida do trabalhador. Oobjetivo deste trabalho foiavaliar se a melhoria da qualidade de vida com implantação de CL influencia a prática dos professores e interfere no processo de ensino aprendizagem. A coleta de dados foi realizada em uma escola de ensino fundamental e envolveu 8 participantesselecionados de acordo com critérios previamente estabelecidos. Os voluntários responderam uma avaliação no início da pesquisa. Após a avaliação inicial, um profissional fisioterapeuta desenvolveu uma rotina de exercícios físicos específicos, que eram aplicados 3 vezes na semana, durante 15 minutos, no meio da jornada de trabalho. Foram realizadas 15 intervenções com a CL e então se aplicou uma reavaliação para tabulação dos resultados. O resultado mostrou diminuição no quadro álgico da maioria dos voluntários. Com isso, os voluntários tiveram maiores motivações em suas práticas profissionais, modificando a didática em sala de aula. Embora necessite de realização da pesquisa com grupo maior e com melhores ajustes no tempo de aplicação da CL, a pesquisa influiu de forma positiva na motivação dos professores e no processo de ensino aprendizagem.
\end{abstract}

Palavras chave: Cinesioterapia Laboral. Atividade Física. Qualidade de Vida. Professores. Ensino Aprendizagem.

\section{THE INFLUENCE OF LABOURCINESIOTERAPIAIN THE LIFE QUALITY OF TEACHERS AND THE TEACHING LEARNING PROCESS}

\begin{abstract}
:
The Labor kinesiotherapy(LK),or labor gymnastics, is a series of physical exercises developed in the workplace. In this context,the aim of this study was to evaluate the improvement of Labor kinesiotherapy deployment with quality of life influences the practice of teachers and interferes with the teaching and learning process. It was developed in a primary school and involved 8 participants selected according previously established criteria and included in a protocol of Labor kinesiotherapy. The volunteers were submitted to anevaluation before the beginning of exercises and anotherone after finish it. The results showed improvements in muscle nociceptive frames. The volunteers became motivated to improve their pedagogical practices, modifying the way of teaching in the classroom. Although requires a new research with a larger group of participants and adjustments in the application schedule of Labor kinesiotherapy, the research influenced positively the teachers providingbenefitsto the teaching-learning process.

Keywords: Kinesiotherapy Labor. Physical Activity. Quality of Life. Teacher. Teaching and Learning.
\end{abstract}

\section{INTRODUÇÃO}

A ideia de melhorar a qualidade de vida dos trabalhadores não é recente e deve ser entendida não apenas como forma de tratamento de doenças, mas também as estratégias de prevenção de adoecimentos (GONÇALVES, 2011). Atualmente, devido à revolução tecnológica em que as pessoas 
convivem, algumas classes profissionais estão mais expostas a adoecer devido à sobrecargaimposta pelo trabalho. Diante disso, mecanismos que amenizem os elementos que causam doenças ocupacionais devem sersempre estudados e implantados (LIMA, 2003).

O ser humano necessita ter uma boa condição em seu ambiente de trabalho. A Organização Mundial de Saúde e do Trabalho (OMST) reconhece que algumas classes profissionais, apresentam desordens clínicas e patologias provenientes do trabalho (MARTINS e BARRETO, 2007). Isto ocorre devido às especificidades ocupacionais que alguns trabalhadores estão inseridos.

A categoria profissional dos professores está incluída dentro do grupo de risco para desenvolvimento de queixas álgicas e doenças relacionadas ao trabalho (CARDOSOet al, 2009). Além disso, esta categoria profissional é uma das que mais sofrem com o estresse e outras síndromes decorrentes da baixa qualidade de vida no trabalho (LIPP e ROCHA, 1996).O risco é elevado devido àsmúltiplas funções desenvolvidas pelos profissionais da educação (professores). O professor, além de ensinar,participa da gestão e do planejamento escolar,se estende às famílias e à comunidade, prepara aulas e corrige provas além de outras atividades pertinentes a função.

O papel do professor extrapolou a mediação do processo de conhecimento do aluno. Ampliou-se a missão do profissional para além da sala de aula a fim de garantir uma articulação entre a escola e a comunidade (GASPARINI, BARRETO e ASSUNÇÃO, 2005). Como consequência disto, o desgaste psicofísico do profissional aumentou muito.

Uma das principais queixas de alteração na saúde dos professores refere-se adoresmusculoesqueléticas (DME). As DME são desconfortos envolvendo músculos, ossos, articulações, tendões, ligamentos, bursas, fáscias musculares, tecido conjuntivo, cartilagens e aponeuroses (RIBEIRO, 2009). AsDME podem comprometer a atividade laborativa, com redução do desempenho na sala de aula eaté levar ao afastamento das atividades profissionais (CARDOSOet al, 2011). Alguns fatores do trabalho docente que favorecem o adoecimento osteomuscular são: longo tempo em postura em pé; carregamento de materiais didáticos com postura inadequada ou sem controle de peso dos materiais, mobiliário inadequado para o profissional, correção de provas e exercícios por longos períodos em posturas inadequadas; movimentação de membros e troncode forma incorreta e com sobrecarga nas articulações; elevação de membros superiores e extensão da coluna cervical para escrever no quadro negro;elevada carga horária de aulas semanais; elevado número de turmas; grande número de alunos por turma; inexistência de programas de orientações e qualidade de vida na escola (RIBEIRO et al, 2011). 
A preocupação pela manutenção de saúde do trabalhador é discutida e necessária em todos os setores de trabalho. Por isso, cada vez mais surgem iniciativas visando proporcionarmelhor qualidade de vida dos funcionários nas diferentes atividades econômicas (LIMA, 2003). Os postos de trabalho não podem ser um campo de desenvolvimento de patologias e angústias,e sim, um local onde o profissional possa desempenhar sua tarefa de forma produtiva, agradável e segura (MARTINS e DUARTE, 2000).Há diferentes programas de qualidade de vida nas empresas, diretamente no local do trabalho, visando à saúde do trabalhador. Um exemplo destes programas é aimplantação de ginástica laboral (GL), também conhecida como cinesioterapia laboral (CL)(GRANDE et al, 2011).

A CLpode ser compreendida como uma série de exercícios físicos elaborados durante o expediente e no próprio ambiente de trabalho (PLETSCH, 2001). O intuito dos exercícios é aliviar as estruturas mais utilizadas durante a execução de suas funções e ativar as partes corporais que não são requeridas (GRANDE et al, 2011). Assim, a CL promove adaptações fisiológicas, físicas e psíquicas, por meio de exercícios dirigidos e adequados para o trabalhador no posto ambiente de trabalho. As adaptações físicas incluem a melhoria da flexibilidade, mobilidade articular e postura. As adaptações fisiológicas incluem estímulos para aumentar a temperatura corporal, tecidual e favorecer a circulação sanguínea. As adaptações psicológicas incluem a integração entre os colegas de trabalho e com os superiores, altera a rotina no setor, gera um momento de descontração, convívio saudável e troca de conhecimentos sobre assuntos diversos e do trabalho em equipe (LIMA, 2003).

A execução daCL no local de trabalho evita o deslocamento dos trabalhadores (DIAS, 1994) e permite maior participação dos colaboradores. A CL é uma forma eficaz de levar os trabalhadores à conscientização sobre a importância da prática da atividade física regular, além de outros hábitos de saúde (ALVAREZ, 2001). O programa de CL deve ser aplicado de maneira diversificada, criativa e inovadora. Por isso deve ser desenvolvido por profissionais competentes, que podem ser fisioterapeutas ou educadores físicos (MILITÃO, 2001).

A CL pode ser classificada como (i) Compensatória, quando realizada no meio do turno de trabalho; (ii) Preparatória ou de aquecimento, quando realizadaantes da jornada de trabalho; (iii)Relaxamento, quando realizada em qualquer momento do turno de trabalho com intuito de relaxar estruturas e membros envolvidos para execução das tarefas do trabalho (LIMA, 2003; MILITÃO, 2003).

Em todos os ambientes laborais, para obter um bom rendimento, os trabalhadores devem manter a saúde íntegra. Desta forma, 
não pode ser diferente para os profissionais da educação. Muito se fala em desfazer a tríade dor-desprazer-trabalho docente vivida nos últimos anos (BARROS e LOUSADA, 2007), mas classe dos professores ainda está aquém de receber atenção em se tratando de qualidade de vida. Para educação de qualidade, depende-se muito do professor, e para o professor poder desempenhar bem a sua função, precisa manter a saúde.

Diante deste cenário, buscou-se desenvolver um programa dequalidade de vida dos docentes com a implantação prática de cinesioterapia laboral no ambiente escolar. O objetivo principal do trabalho foi avaliar se a melhoria da qualidade de vida com implantação de CL influenciaa prática profissional dos professores e repercute no processo didático.

\section{MATERIAIS E MÉTODOS}

A pesquisa de natureza aplicada (GIL, 2002), foi realizada com os professores de uma Escola pública das séries iniciais do Ensino Fundamental, no município de Rio Branco do Sul, região metropolitana de Curitiba, estado do Paraná. A amostra foi selecionada de forma probabilística aleatória simples, com participação voluntária. Para participar da pesquisa, foram elencados como critérios de inclusão: ser professor atuante em sala de aula com formação superior completa de ambos os sexos; estar trabalhando na instituição de realização da pesquisa há pelo menos um mês, cumprir no mínimo 20 horas semanais e possuir faixa etária entre 22 a 60 anos. Como critérios de exclusão foram determinados: Possuir contraindicação à realização de movimentos por patologias ou cirurgias;apresentar-se inapto pela avaliação inicial realizada pelo pesquisador; ter jornada de trabalho diferente da função de professor em outro turno; desistir durante o programa de pesquisa por interesse próprio ou por força maior; possuir idade inferior ou superior ao alvo da pesquisa; ser transferido para outra instituição de trabalho ou para outro cargo na escola fora da sala de aula e; não completar o número de intervenções propostas na pesquisa. Antes de iniciar a pesquisa todos os possíveis voluntários foram orientados pelo pesquisador sobre as etapas da pesquisa. Paraparticipar deveriam assinar o Termo de Consentimento Livre e Esclarecido (TCLE), respeitando a autonomia do indivíduo, conforme resolução 196/96 do Conselho Nacional de Saúde (CNS). Após obtenção do TCLE, o pesquisador fez uma consulta inicial para conhecer o estado de saúde de cada participantee arealização de anamnesee testes específicos para verificar qualquer impossibilidade ou contra indicações do tratamento de Cinesioterapia Laboral. No final da consulta, o participante que estava apto a participar da pesquisa, respondia a avaliação inicial (AI), desenvolvida pelo pesquisador especificamente para este trabalho. A avalição Inicial foi composta de 
15 itens, contemplando: (1) nome; (2) sexo; (3) faixa etária; (4) carga horaria semanal; (5) tempo de atuação no magistério de Educação Infantil; (6) número de alunos por classe; (7) se o participante realizava atividades físicas frequentemente; (8) se sentia dores musculares após ministrar as aulas; (9) quais as partes corporais que possuíam maiores dores/tensões após as aulas; (10) se ficaram em afastamento da sala devido às atividades laborais na educação; (11) se deixaram de realizar alguma atividade lúdica ou forma de ensinar por sentir-se indisposto e/ou para não sentir sobrecarregado após a aula; (12) se já haviam participado de campanhas ou outros projetos de melhoria de qualidade de vida e saúde na escola; (13) se já houve momentos que pensaram em desistir da profissão devido aos poucos investimentos na qualidade de vida; (14) se consideravam necessários implantar exercícios físicos e outras técnicas visando à melhoria de qualidade de vida durante o turno do trabalho e; (15) auto avaliação de como classificavam a sua saúde física perante o trabalho (excelente, boa, regular, ruim/péssima). Como todos os voluntários passaram pelo pesquisador em uma sala individual devido ao sigilo, não houve risco de um voluntário manipular as respostas de outro. Posteriormente aos procedimentos de avaliação inicial, elaborouse um cronograma para realização de exercícios terapêuticos em grupos, enfatizando os pontos dolorosos relatados na consulta e avaliação inicial dos voluntários, adaptados de uma sequência de exercícios propostos por Anderson (2003). A aplicação da CL aconteceu entre os meses de outubro e dezembro de 2012, com frequência de três vezes por semana (segundas, quartas e sextas), no período da tarde, durante o intervalo dos alunos, com duração de 15 minutos cada sessão. Os professores tiveram 15 encontros de aplicação de CL. Havia uma lista de presença para confirmar a participação de cada voluntário nas sessões. Finalizando os encontros, os voluntários foram convidados a responder a avaliação Final (AF), composta de 3 itens, por meio da qual buscou-se saberse (1) o voluntário observou melhora na sua motivação pessoal e profissional após iniciar o programa de cinesioterapia laboral, (2) sentiu melhora corporal durante a aplicação da cinesioterapia laboral, com redução de dor/tensão nos pontos relatados antes do inicio da pesquisa, (3) recomendariam o programa para outros professores. Os dados foram tabulados usando o calculo de percentual simples com o programa Microsoft-Excel ${ }^{\circledR}$. A seguir, serão descritosos resultados da pesquisa e a discussão com outros dados disponíveis na literatura.

\section{RESULTADOS E DISCUSSÃO}

Foram incluídos na pesquisa 10 voluntários, porém um foi transferido de escola e outro faltou durante o programa 
devido a atestado médico por doenças infectocontagiosas e não completou o número de sessões propostas, sendo ambos excluídos. Portanto, finalizaram a pesquisa 8 voluntários. Embora pudessem participar da pesquisa ambos os sexos, todos os participantes foram do sexo feminino, o que correspondeu $100 \%$ dos voluntários. Os voluntários selecionados possuíam diferentes faixas etárias, variando de 23 anos a 55 anos.

Em relação à carga horária semanal, todos os voluntários relataram cumprir 20 horas semanais nesta mesma escola, pois trata de uma instituição de ensino das séries iniciais do ensino Fundamental e todas possuem apenas um padrão no concurso do município. Quanto ao número de alunos na sala de cada voluntária, variou entre 25 a 30 alunos. O número de alunos deve ser levado em consideração em uma pesquisa que relaciona qualidade de vida e educação, pois segundo Cardoso et al(2009), as DME foram mais prevalentes em professores com turmas superiores a 30 alunos, pois sugerem que o aumento do número de turmas e de alunos acarreta, para o professor, acréscimo em suas atividades.

Em relação ao questionamento sobre a prática de exercícios físicos de forma regular, apenas uma voluntária respondeu que executa exercícios físicos regulares, o que representa $12,5 \%$, enquanto que $87,5 \%$ negaram praticar exercícios físicos.
Pelos dados apresentados nesta pesquisa, verificou-se que a maioria dos professores não realiza atividade física. Neste caso, a aplicação cinesioterapia Laboral no ambiente escolar pode ser aplicada com o intuito de minimizar os impactos negativos oriundos do sedentarismo na vida e na saúde do professor (CARVALHO, 2003).Sabe-se que é importante desde cedo prevenir problemas de saúde.Pressi e Candotti (2005) reforçam que a inserção da CL durante a rotina de trabalho nas empresas pode apresentar resultados positivos, melhorando a produtividade e a disposição para o trabalho, melhoria da qualidade de vida e conscientização corporal.

Soares, Assunção e Lima (2006) afirmam que a CL pode ser eficaz para prevenir e combater os distúrbios físicos e emocionais na saúde dos trabalhadores, tais como estresse, lombalgias e doenças que o trabalho pode acarretar. Assim, a CL está indicada para os professores, pois na pesquisa realizada, todos os voluntários responderam que já sentiram dor após ministrar uma aula. Quanto aos segmentos corporais citados onde apresentaram maiores dores, foram os mais diversos, sendo que $62,5 \%$ relataram histórico de dor em região dos membros superiores (ombros e/ou Braços), 25\% assinalaram ter sofrido de dores em membros inferiores (pernas) e $12,5 \%$ relataram dores na coluna (cervical, dorsal/torácica e lombar), conforme se observa no gráfico 1 . 


\section{Gráfico 1 - Percentual de voluntárias com os} segmentos corporais que sentem maiores dores.

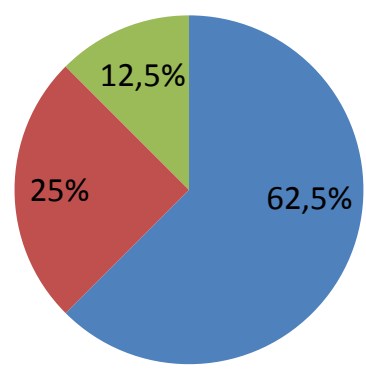

Membros

superiores

Membros

inferiores

Coluna

(Cervical, torác ica e Lombar)

Os dados mostrados em relação às partes corporais com maiores dores corroboram com estudos de Cardoso et al (2009), os quais descreveram em seus estudos que os professores investigados referiam elevadas prevalências de dor musculoesquelética em braços e ombros (membros superiores), pernas e coxas (membros inferiores) e na coluna dorsal. Ademais, um voluntário assinalou que já teve histórico de afastamento do trabalho docente pela atividade ocupacional. Alguns malefícios provenientes da atividade laboral tais como o estresse, más posturas, mobílias inadequadas e movimentos repetitivos facilitam o aparecimento de doenças relacionadas ao trabalho e resultam no afastamento de grande percentual de trabalhadores de suas funções, sendo a principal causa de indenizações de auxílio doença e a terceira causa de aposentadoria por invalidez (CANDOTTI, STROSCHEIN E NOLL, 2011). O impacto social e econômico

ocasionado pelos distúrbios osteomusculares ocupacionais vêm crescendo de forma preocupante e mecanismos que amenizem este impacto deve ser implantados. Bergamaschi, Deutsch e Ferreira (2002) relatam que entre os benefícios da cinesioterapia Laboral para a empresa, podese observar a diminuição do absenteísmo, rotatividade de funcionários e afastamentos. Em outras palavras, a aplicação do protocolo de CL pode ser uma alternativa para reduzir os afastamentos. Diante deste cenário, justifica-se a necessidade de maiores pesquisas e investimentos na área de promoção e prevenção à saúde, incluindo a CL, visando proporcionar a qualidade de vida dos docentes atuantes em sala e também às demais pessoas envolvidas do processo de ensino-aprendizagem como a coordenação, supervisão pedagógica, direção, entre outros.

A atividade laboral do profissional professor é dinâmica. Na sala de aula, o professor adota uma determinada postura e enquanto prepara as aulas adota outra postura, normalmente em frente ao computador. Nem sempre esses profissionais possuem uma consciência corporal e postural correta, o que faz aumentar ainda mais as tensões e dores corporais. Os erros posturais e ergonômicos dos postos de trabalhos ampliam o favorecimento e riscos de aparecimentos de dores e lesões. Souza e Joia (2006) endossam o fato de que a atividade física pode ser uma das alternativas na prevenção das doenças em 
pessoas que trabalham sentadas e usuárias de computador. Para esses trabalhadores que adotam posturas inadequadas, os exercícios físicos são fundamentais, porque alongam e relaxam a musculatura tensionada, principalmente os músculos estabilizadores da coluna (paravertebrais), permitindo que haja diminuição da fadiga e aumento do desempenho profissional.

Ao relacionar as dores corporais $\mathrm{e}$ ensino, em um primeiro momento é possível pensar que não tem relação alguma, entretanto, ao sentir dor e tensão, o profissional deixa de realizar determinadas aulas para poupar seu corpo e para evitar dores futuras e assim manter energia. As aulas ficam monótonas, sem nova perspectiva aos alunos, deixando-os desinteressados em aprender. Ao responder a avaliação inicial, no tópico "Você já teve dificuldade de fazer seu trabalho ou já deixou de realizar alguma atividade diferente em sala de aula com os alunos para não sobrecarregar o corpo $e$ evitar dor/tensão?", todas asparticipantes da pesquisa responderam "sim", abrangendo $100 \%$ dos participantes, o que permite verificar que a qualidade de vida atinge diretamente a classe dos professores e por consequência a qualidade das aulas e do ensino.

Embora muito comentado que a atividade física traz melhorias para a saúde, de acordo com os dados desta pesquisa, a classe dos professores ainda não tem incluída no dia a dia tal prática. Por isso, é importante incluir palestras ou simpósios a respeito da manutenção e melhoria da qualidade de vida com prática diária de atividade física no próprio ambiente escolar. Assim, os voluntários foram questionados se já tinham participando ou tiveram orientação a respeito de práticas de exercícios físicos e métodos saudáveis anteriormente à pesquisa na escola. O resultado mostrou que $100 \%$ dos voluntários não receberam nenhuma orientação acerca de prática de exercícios físicos no ambiente escolar anteriormente a esta pesquisa. Em contrapartida, todos os voluntários (100\%) responderam que é importante dedicar alguns minutos no seu turno de trabalho para receber orientações e realizar práticas de exercícios físicos, como a cinesioterapia Laboral, visando uma melhoria qualidade de vida na escola e consequentemente melhora no ensino. Para a questão “Você já pensou em desistir da profissão de professor por não apresentar um bom programa de qualidade de vida aos profissionais?", metade dos voluntários respondeu que sim (50\%), o que indica que a os professores sentem necessidade de programas que priorizem a sua saúde dentro da escola.

O último questionamento da avaliação inicial buscava informações sobre como as voluntárias observam a sua própria saúde, tendo quatro opções de resposta. A maioria das participantes $(62,5 \%)$ classificou a sua 
própria saúde como "boa", enquanto que a minoria respondeu como excelente $(12,5 \%)$. Os outros $25 \%$ consideraram sua saúde regular, portanto não teve nenhum participante que considere sua saúde ruim ou péssima. Pode-se verificar esse percentual ilustrado no gráfico2.

\section{Gráfico 2 - Percentual como as voluntárias classificam o próprio estado de saúde}

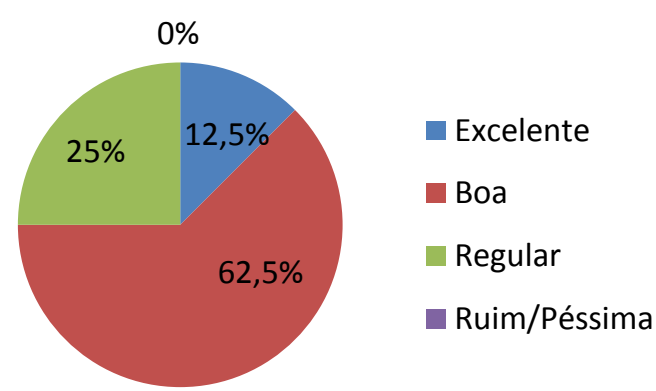

A avaliação final foi composta de apenas três questões. A primeira questão perguntava se o programa de CL influenciou na sua motivação profissional, fazendo com que tivesse aplicado pelo menos uma aula diferente em sala que não tinha trabalhado anteriormente com os alunos para poupar-se fisicamente. Como resultado a essa questão observou-se que $100 \%$ assinalaram a resposta que se sentiram mais motivadas durante o programa e fizeram alguma atividade diferente em sala, podendo ser atividade lúdica, pesquisa de campo ou qualquer outra atividade que utilizasse outra forma didática além da comumente utilizada. Os voluntários relatavam que sentiam maior ânimo para trabalhar com os alunos.

Ainda de acordo com os dados da pesquisa, $87,5 \%$ das voluntárias relataram melhora no quadro doloroso ou em relação atensão muscular. Também relataram melhor disposição durante seu trabalho docente após o início da aplicação da CL. Não foi questionado se as dores sumiram ou se apenas diminuíram, visto que o presente estudo trabalho não tinha por objetivo quantificar o nível de alívio da dor, podendo isso seguir em uma linha de estudo futura. Questionou-se apenas se houve ou não melhora da dor/tensão e consequentemente melhor disposição no seu dia a dia e no trabalho. Estes achados estão de acordo com Pinto (2003). Este autor relata que os benefícios físicos da cinesioterapia laboral são algo incontestável, uma vez que, toda e qualquer atividade física devidamente aplicada é vantajosa. Moura-Junior e Dechechi (2010)também relatam que a ginástica ou cinesioterapialaboral contribui para o melhor rendimento profissional dos funcionários, pois ao praticar exercícios físicos adaptados às necessidades de cada trabalhador, toda a empresa sai beneficiada, poisdiminuem os afastamentos dos trabalhadores e reduzem-se as doenças causadas pelos movimentos repetitivos, pela postura ou estresse, como é o caso do professor.

Para Souza e Jóia (2006), a prevenção do sedentarismo e das doenças 
cardiovasculares já seriam motivos suficientes para afirmar que a cinesioterapia laboral, sob caráter de atividade física, produz melhor qualidade de vida. No entanto, vale ressaltar que o indivíduo não deve ser forçado a fazer o que não quer, ou seja, se o funcionário não quiser aderir à cinesioterapia laboral, sua decisão deve ser respeitada, para que o mesmo não sofra pressão psicológica e apresente rendimento contrário ao esperado. Por isso, para esta pesquisa todos os professores, dentro dos critérios de inclusão e exclusão foram convidados a participar, mas caso em algum dia não quisesse realizar as atividades, de forma alguma se insistia para a sua participação. No entanto, em todos os dias da aplicação do protocolo todos os participantes estavam presentes, demonstrando interesse por parte dos voluntários em fazer as atividades.

A aplicação de CL á algo comum em setores industriais, porém em ambiente escolar e com os professores, é algo inovador. Muito se fala em qualidade de educação, mecanismo de melhoria na escola, porém sãopoucos os projetos desenvolvidos visando à qualidade de vida do professor, que é peça chave no processo de ensino-aprendizagem. Assim, por se tratar de aplicação de CL no ambiente escolar, onde comumente não tem esta rotina e os profissionais não estão totalmente habituados com esta modalidade, esta pesquisa preocupou-se em investigar se os voluntários indicariam essa forma de prevenção para seus colegas, mesmo que para outros níveis de ensino. O resultado foi unânime, sendo que $100 \%$ das voluntárias indicariam a realização do protocolo para os colegas. Com isso pode-se verificar que mesmo a técnica alterando o ciclo normal do dia a dia da escola, os participantes concordam com a execução de CL e indicam aos seus colegas de profissão, certamente por sentirem melhora corporal no dia a dia. Todos os participantes e também a direção da escola gostariam que o programa continuasse, pois os resultados foram muito vantajosos, porém para dar continuidade dependeria de aprovação da gestão municipal e contratação de profissionais para o estabelecimento, o que não era foco desta pesquisa em buscar recursos junto aos órgãos responsáveis. Entretanto, com os dados apresentados a escola pode iniciar um processo de negociação e mostrar o quanto a inserção desta forma de projeto beneficia os professores, os alunos e também a sociedade, pois em se tratando de melhoria de educação todos ganham.

\section{CONCLUSÃO}

Após análise da literatura atual e da aplicação prática do protocolo de cinesioterapia laboral desenvolvida diretamente no local de trabalho, os resultados permitiram inferir que a intervenção da CL melhorou a motivação pessoal e profissional 
dos envolvidos na pesquisa, pois todas as voluntárias responderam que aplicaram pelo menos uma aula diferente durante o programa de cinesioterapia laboral, o que facilita a interação professor-aluno e favorece a troca de conhecimentos em sala de aula.

Nesse trabalho também foi possível observar que os professores sentem dores em diferentes partes corporais após aplicar suas aulas. Após a aplicação da CL, a maioria relatou melhora $(87,5 \%)$ o que leva a crer que a implantação desses programas de qualidade de vida e atividade física nas escolas traz melhora corporal do professor, pois anteriormente à pesquisa, todos os professores relataram dor em alguma região corporal. Além desse fato, a maioria consideravaa sua própria condição de saúde em estado regular, assim, a diminuição das algias corporais permitiu concluir que os professores precisam de medidas preventivas em sua saúde para ter melhor influencia no processo educativo durante as suas aulas. Adicionalmente, o programa de cinesioterapia laboral é recomendado por todas as voluntárias aos demais colegas.

Com os resultados apresentados verificou-se que é importante inserir alguns minutos para a prática de exercícios físicos durante o turno dos educadores e professores, para evitar a fadiga corporal e a desmotivação pessoal. Quando o profissional sente desconfortos no corpo deixa de aplicar metodologias diversificadas, havendo diminuição no processo de ensino aprendizado dos alunos. Com a aplicação da CL, a motivação dos profissionais apresentou melhora e todas as voluntárias aplicaram novas abordagens metodológicas durante as aulas, o que aproxima professores e alunos e, portanto, houve a motivação e também a melhoria da qualidade de aprendizado.

Por ser feito em pequena escala, podese destacar que o projeto deve ser continuado. Entretanto, para trabalho futuros, recomendase mensurar o nível de melhora álgica dos professores, realizar o protocolo com maior número de voluntários, identificar um horário entre o turno real de trabalho dos docentes e não no horário de intervalo e ainda continuar o protocolo por um período maior de tempo, para que os benefícios adquiridos sejam mantidos e aprimorados ainda mais com o decorrer do tempo de aplicação.

\section{REFERÊNCIAS}
ALVAREZ, Bárbara Regina. O Papel da Ginastica Laboral nos programas de promoção da saúde. In: CONGRESSO BRASILEIRO DE ATIVIDADE FÍSICA E SAÚDE, 3.; Florianópolis: UFSC, 2001. p. 17-18.

ANDERSON, Bob. Alongue-se. 23. ed. rev. e atual. São Paulo, SP: Summus, 2003.

BARROS;Maria Elizabeth, LOUZADA, Ana Paula; Dor-Desprazer-Trabalho Docente: Como Desfazer essa tríade? Revista Psicol. USP, out./dez. 18(4), 13-34 SP 2007.

BERGAMASCHI. Elaine Cristina; DEUTSCH, Silvia; FERREIRA, Eliane Olito. 
Ginástica Laboral: Possíveis inplicações para as esferas física, psicológica e social. Revista Atividade Física \& Saúde. v.7, n.3, 2002.

CANDOTTI. Claudia Tarragô, STROSCHEIN. Rosemeri, NOLL. Matias; Efeitos da ginástica Laboral na dor nas costas e nos hábitos posturais adotados no ambiente de trabalho. Rev. Bras. Ciênc. Esporte, Florianópolis, v. 33, n. 3, p. 699-714, jul./set. 2011.

CARDOSO, Jefferson Paixão;

ARAUJO.Tânia Maria

de;CARVALHO.Fernando

Martins; OLIVEIRA.Nelson Fernandes

de;REIS.Eduardo José Farias Borges dos;Aspectos psicossociais do trabalho e dor musculoesquelética em professores. Cad. Saúde Pública, Rio de Janeiro, 27(8): 1498-1506, ago, 2011.

CARDOSO, Jefferson Paixão; RIBEIRO, Isadora de Queiroz Batista; ARAÚJO Tânia Maria de; CARVALHO, Fernando Martins; REIS, Eduardo José Farias Borges dos;Prevalência de dor musculoesquelética em professores. Ver BrasEpidemiol; 12(4): 604-14, 2009.

DIAS, M. F. M. Ginástica laboral: Empresas gaúchas têm bons resultados com ginástica antes do trabalho. Revista Proteção, V. 6, n. 29. p. 24 - 25, mai. 1994.

GASPARINI, Sandra Maria. BARRETO, Sandhi Maria. ASSUNÇÃO, Ada Ávila. O professor, as condições de trabalho e os efeitos sobre sua saúde. Revista Educação e Pesquisa, São Paulo, v. 31, n. 2, p. 189-199, maio/ago. 2005.

GIL, Antonio Carlos.; Como elaborar projetos de pesquisa. 4. Ed, Atlas, SP, 2002.

GONÇALVES, Aguinaldo; Conhecendo e discutindo saúde coletiva e atividade física. Guanabara Koogan, RJ, 2011.
GRANDE, Antonio José. LOCH, Mathias Roberto. GUARIDO, EvanilAntonio. COSTA, João Bruno Yoshinoga. GRANDE, Gabriela Claudino. REICHERT, Felipe Fossati; Comportamentos relacionados à saúde entre participantes e não participantes da ginástica Laboral. Rev. Bras. Cineantropom. Desempenho Hum: 3(2): 131-137, 2011.

LIMA;Valquíria de,Ginástica laboral: atividade física no ambiente de trabalho. $1^{\mathrm{a}}$ edição, Ed. Phorte, SP, 2003.

LIPP, Marilda; ROCHA, João Carlos. Stress, Hipertensão arterial e qualidade de vida. 2.ed. São Paulo: Papirus, 1996.

MARTINS, Gizele de Cássia; BARRETO, Selva Maria Guimarães. Vivências de ginástica laboral e melhoria da qualidade de vida do trabalhador: resultados apresentados por funcionários administrativos do instituto de física da Universidade de São

Paulo(Campus São Carlos) Motriz, Rio Claro, v.13 n.3 p.214-224, jul./set. 2007. MARTINS, Caroline de Oliveira. DUARTE, Maria de Fátima da Silva; Efeitos da Ginástica laboral e servidores da reitoria da UFSC. Rev. Bras. Ciên. e Mov. v.8 n. 4 p. 0713, DF, 2000.

MILITÃO, Angeliete Garcez.; Influência da ginástica laboral para a saúde dos trabalhadores e sua relação com os profissionais que a orientam. Dissertação de Mestrado apresentada ao Programa de Pósgraduação em Engenharia de Produção da Universidade Federal de Santa Catarina, UFSC, Florianópolis, SC, 2001.

MOURA-JUNIOR, Anibal de; DECHECHI, Clodoaldo José; Benefícios da Ginastica Laboral em empresas: Uma Revisão de Literatura. Revista Hórus. V. 4. n. 1, 2010.

PINTO. Alexandre Crespo Coelho Da Silva; Ginástica laboral aplicada à saúde do cirurgião dentista um estudo de caso na Secretaria Municipal de Saúde de Florianópolis - SC. Dissertação de Mestrado em Engenharia de Produção. Programa de 
Pós-Graduação em Engenharia de Produção, Universidade Federal de Santa Catarina.

UFSC, SC; 2003.

PLETSCH, Celei. Ginástica Laboral: Um estudo de caso em Marechal Cândido

Rondon-PR. Caderno de Educação Física. v3. n2. p 49-65, 2001.

PRESSI, Ana Maria S.; CANDOTTI, Cláudia Tarrago; Ginástica Laboral. São Leopoldo: UNISINOS, 2005.

RIBEIRO, Isadora de Queiroz Batista. ARAÚJO, Tânia Maria de. CARVALHO, Fernando Martins. PORTO, Lauro Antonio. REIS. Eduardo José Farias Borges dos; Fatores Ocupacionais associados à dor Musculoesquelética em Professores. Revista Baiana de Saúde Pública. v.35, n.1, p.42-64 jan./mar. 2011

RIBEIRO, Isadora de Queiroz Batista; Fatores ocupacionais associados à dor músculo-esqueléticas em professores. Dissertação apresentada ao Programa de Mestrado em Saúde, Ambiente e Trabalho da Faculdade de Medicina da Universidade Federal da Bahia, UFBA, BH, 2009.

SOARES, Raquel Guimarães; ASSUNÇÃO, Ada Ávila ; LIMA, Francisco de Paula Antunes.Searching for elements at work that could explain the low attendance to a labor gymnastics program.Revista Brasileira de Saúde Ocupacional, São Paulo, v. 31, n.114, p.149-60, jul./ago. 2006.

SOUZA, Bianca Cristina Conceição de.

JÓIA, Luciane Cristina; Relação entre

Ginástica Laboral e Prevenção das Doenças Ocupacionais: Um Estudo Teórico. Faculdade São Francisco de Barreiras. Barreiras.SP. 2006. Disponível em:

http://www.fisiobrasil.com.br/main.asp?link= materias\&grup $=43 \#[$ AbreEmDIV]ajax.asp?li $\mathrm{nk}=$ amateria\&id=289 Acesso em agosto/2012. 